$$
\text { "tmcs-R-Sipos" — 2009/6/2 — 23:53 — page } 69-\# 1
$$

\title{
Apollonius' problems in grammar school
}

\author{
ElviRA Ripco Sipos
}

\begin{abstract}
In this work there are ten problems of Apollonius listed with illustrations and solution possibilities including students' solutions, too. Usually, it is rather difficult for students to grasp the essence of these problems with the use of traditional means, bows and rulers, but the use of computers offers higher accuracy.
\end{abstract}

Key words and phrases: Apollonius' problems, inversion, construction, DGS, visualization.

ZDM Subject Classification: G60, G90.

\section{Introduction}

I'm a geometry teacher in Bolyai Grammar school in Senta, Serbia. This is a unique school in Serbia, because the teaching language is Hungarian, but the school is accredited as a special mathematical secondary school with programs given by the Ministry of Education of Serbia. The Bolyai Secondary Grammar School and Dormitory for Gifted Students is unique in Vojvodina in respect that it targets the talented students of the region, specializes in natural sciences and mathematics and offers Hungarian as the language of instruction. The school was founded under the name of Secondary Grammar School Specialized in Natural Sciences and Mathematics for Gifted Students on April 22, 2003. Besides Hungarian, the curriculum comprises of English, Serbian language and biology, history, geography, physics, chemistry and informatics, and there are four mathematical subjects as well.

Copyright (C) 2009 by University of Debrecen 


$$
\text { "tmcs-R-Sipos" — 2009/6/2 — 23:53 — page } 70-\# 2
$$

(1) Analysis and Algebra in every grade with 4 lessons per week;

(2) Geometry in first and second grade with 4 lessons per week

(3) Linear algebra and analytical geometry in third grade with 3 lessons per week;

(4) Probability and statistics in fourth grade with 2 lessons per week.

The graduate exam includes written exams from Hungarian language, Analysis and Algebra, and the third subject is chosen freely from Geometry, Physics and Informatics. The students in the "Bolyai" school have to pass a preliminary exam, which is organized for the pupils in elementary school, eighth class (that means 14 years old), in June every year. This exam contains very difficult tasks from elementary mathematics, but using higher methods. That is why teachers of mathematical subjects of the school keep preparing lessons every winter and spring from January to May. Working with potential students of the school teachers get knowledge about their skills and can help to refill gaps in the methods of problem solving, in deepening the knowledge, to understand the definition of the concept. There are 20 legal places to take students to the first class; this is the right of the school "Bolyai". There are much more beginners on the first meeting, but on the end there are about 50-60 pupils on the preliminary exam. The conditions of this exam provide that now there are 22 students in the first class, 20 in the second, 19 in the third and 14 in the fourth form. The first generation graduated last year, graduate exams gave excellent results, and average marks were 4,33 from geometry, and 4,5 from analysis and algebra. All of them were admitted to the chosen universities in Serbia and Hungary and won scholarship to their studies. It is very important to develop cognitive attitude of the students in Geometry I, to help the development of the thinking skills, the sense of the space, and the approach to the modern sciences. 15 year-old students have undeveloped mathematical thinking; they do not realize the problem solving thinking because they do not have experience in solving such tasks. Geometry gives great help in effect to carry out given problems. For more than four years I have been teaching Geometry 1 at Bolyai Grammar school Senta for gifted pupils specializing in mathematics and natural sciences. They have 144 geometry lessons per year and I teach every 4th lesson; that means 25 percent of the lessons, which are held in the IT classroom. 75 percent of the lessons are "classical" geometry, using paper in "classical" constructions with pencil, rulers and bows, because they have to develop their manual skills.

The program was built up following the introduction of basic geometrical knowledge. There are: 
"tmcs-R-Sipos" — 2009/6/2 — 23:53 — page 71 — \#3

(1) Isometric transformations: rotation, reflection, symmetry, translation;

(2) Dilatation, symbol $H_{O, k}$, where $O$ is the center of the dilatation, $k$ is the ratio;

(3) Inversion, symbol $\Psi_{i}(O, r)$, where $O$ is the center and $r$ is the radius of the inversion circle $i$.

As the example of using inversion to a circle there are Problems of Apollonius, their constructions, discussion, analyze and proof. Those who have some experiences in construction with the chalk on the whiteboard with bows and rulers have an idea of how to show and prove these interesting but difficult problems to their own students. The emphasis is on convincing instead of proving. A great help in the visualization is some DGS as "Cinderella", "Geogebra", "Geonext" or "Euklides".

As one can read in [7], that: "More sophisticated computer environments have been designed in recent years which take advantage of flexible computer interfaces Geometric software ... allows figures to be drawn with specific relationships defined, such as a given point must always lie at the midpoint of a given line-segment, or be constrained to lie on a given circle. Then the figure may be pulled around inactively retaining all defined constrains to investigate possible consequent relationships.... Such software may be used to gain enactive visual support in conjecturing and testing geometric theorems, enabling students to take an active part in the construction of their own knowledge, though once again, the formal proof structure of geometry will need separate consideration." We got a licence from László István in 2004 to use the last one, Euklides. We construct particular solutions, and by moving the basic elements we can show every solution to the problem.

Constructional tasks are chosen to develop motorical skills, accuracy and to find out the differences between conditions, assumptions and consequences. My aim is to grow up the problem solving thinking by visualizations on Computers, and to work out the recognition of the axiomatically built geometry in the plane. Visualization helps students with weaker achievement to understand the problems, but the special talented students enjoy the probabilities of the animation. They need this kind of visualizations, as well, but with larger, more difficult problems to solve on their level. "It is so beautiful to find out special cases by dynamic movement of basic elements,measuring elements of the last figure"- pupils say. It is interesting to the teacher, as well, when pupils find more methods of solutions of the given task, different from the known solutions. As David Tall says in [7]: "With a computer tool capable of carrying out some of these processes, a 


$$
\text { "tmcs-R-Sipos" — 2009/6/2 - 23:53 - page } 72 \text { - \#4 }
$$

new form of learning becomes feasible in which the individual can concentrate on constructing mental relationships that are important to conceptualization, whilst the computer carries out the routine algorithms. It is the task of educator to provide appropriate environments to focus on selected mathematical concepts or processes whilst suppressing routine algorithms carried out by the computer, to enable the learner to make selected mathematical constructions. i term this the principle of selective construction." Thats why my choice was the most difficult construction, Problems of Apollonius.

\section{Problems of Apollonius}

Apollonius of Perga about 262 BC - about 190 BC .

Apollonius was a Greek mathematician known as 'The Great Geometer'. His works had a very great influence on the development of mathematics and his famous book Conics introduced the terms parabola, ellipse and hyperbola [4]. Pappus mentions other treatises of Apollonius:

- De Rationis Sectione ("Cutting of a Ratio")

- De Spatii Sectione ("Cutting of an Area")

- De Sectione Determinata ("Determinate Section")

- De Tactionibus ("Tangencies")

- De Inclinationibus( "Inclinations")

- De Locis Planis("Plane Loci")

In Book IV of The Elements, Euclid [2] shows how to construct the circle that passes through three given points, and also how to construct a circle tangent to three given straight lines. Apollonius of Perga subsequently generalized this by showing how to find a circle tangent to three objects in the plane, where the objects can be any combination of points, lines, and/or circles. The most general and difficult case is obviously the case of three circles, which was covered in Book II of Apollonius' "On Tangencies". The basic problem is: Given three objects, each of which may be a point, line, or circle, draw a circle that is tangent to each. 
There are a total of ten cases [5]:

(1) CIRCLE CROSSES THREE GIVEN POINTS $A, B, C$.

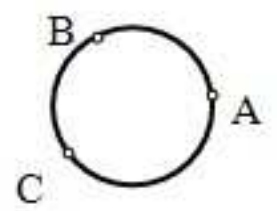

Solution: Three no collinear points $A, B, C$ determine triangle $A B C$, and its circumcircle is the searched circle. Its center is the intersection point of lines of symmetry of edges in triangle $A B C$.

Euclid [2] in the IV. book in proposition 5 states: "And it is manifest that when the center of the circle falls within the triangle, the angle BAC, being in a segment greater than the semicircle, is less than a right angle, when the center falls on the straight line $B C$, the angle $B A C$, being in a semicircle, is right, and when the center of the circle falls outside the triangle, the angle $B A C$, being in a segment less than the semicircle, is greater than a right angle."

Discussion: This problem always has a solution, if the given points aren't collinear points.

(2) CIRCE CROSSES TWO POINTS AND TANGENTS THE GIVEN LINE $A, B, p$

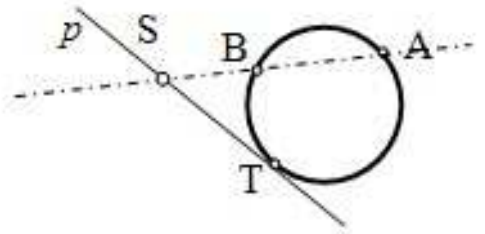

Solution: Let $T$ be the point of tangency of the given line $p$ and the circle, and let $S$ be the intersection point of lines $A B$ and $p$. Then $S A \cdot S B=S T^{2}$, because the product of the lengths of the whole secant and the part outside the circle is the same for every secant, and equal to the square of the tangential segment. This segment $S T$ is constructible with right triangle and its' own altitude. First of all construct point $S$, and then point $T$ on line $p$. Let line $n$ be the perpendicular to line $p$ across point $T$, and let $s_{A B}$ be the bisector of segment $A B$. The intersection point of lines $n$ and $s_{A B}$ is the center of the circle. 
Discussion: Let $h$ be the distance of point $A$ from the line $p$, and similarly $H$ is the distance of point $B$ from line $p$ and $d=H-h$. This problem has solutions if and only if $0<h<H, d>0$.

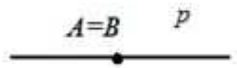

i) double points

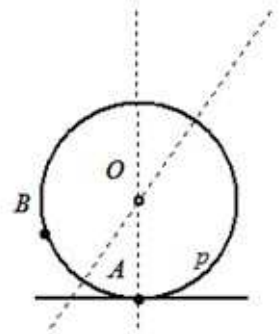

iv) one solution
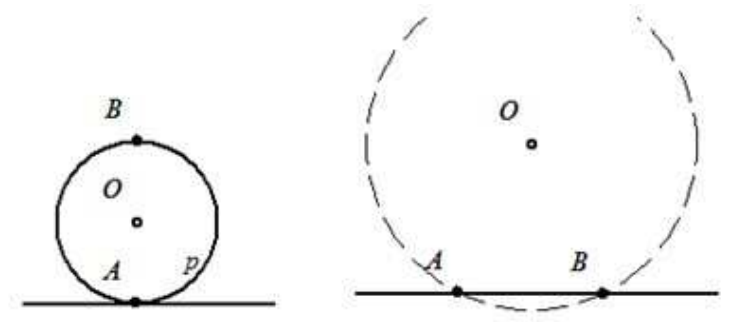

ii) circle with diameter $\mathrm{H}$

iii) no solution

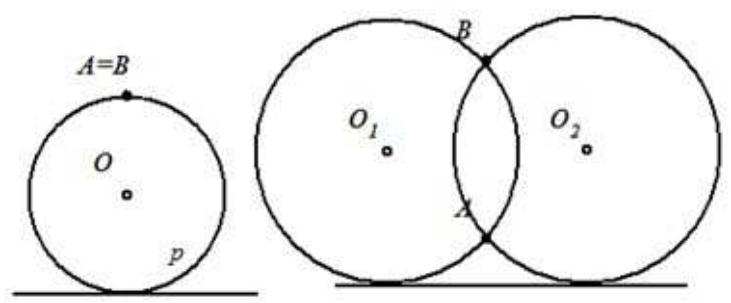

v) circle with diameter $\mathrm{H}$

(3) CIRCLE ACROSS TWO POINTS TANGENTS THE GIVEN CIRCLE $A$, $B, l$

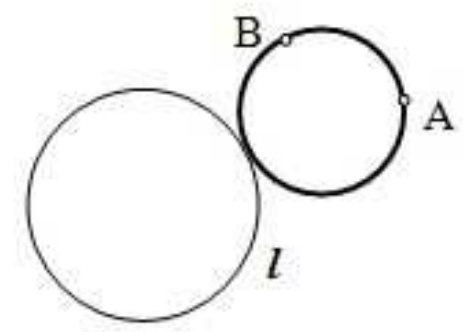

Solution: Use inversion $\psi_{i}(A, r)$ with center A and arbitrary radius r. Let $l^{\prime}$ be the image of circle $l$ in that inversion, so this is a circle, if $A$ isn't on circle $l$, and $B^{\prime}=\psi_{i}(B)$. Construct tangencies $k_{1}^{\prime}$ and $k_{2}^{\prime}$ across point $B^{\prime}$ to circle $l^{\prime}$. These lines $k_{1}^{\prime}$ and $k_{2}^{\prime}$ are images of the demanded circle. Using $\psi_{i}$ again there are two solutions of the given problem, because inversion is involution 
( $\psi_{i}^{2}$ is identical transformation).

The phases of the construction:
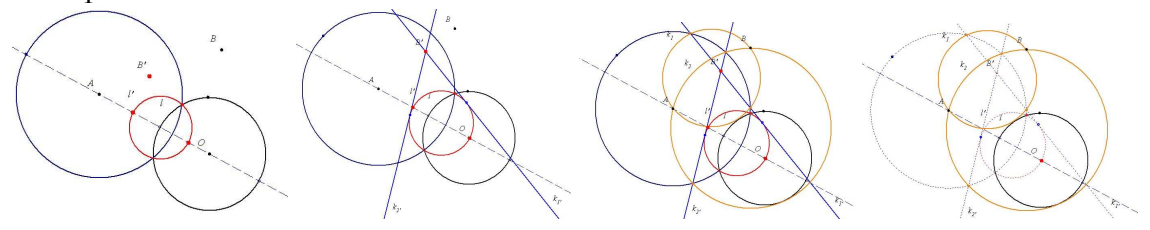

Discussion:

i) If $A$ and $B$ are two different points outside of the given circle, then there are two solutions.

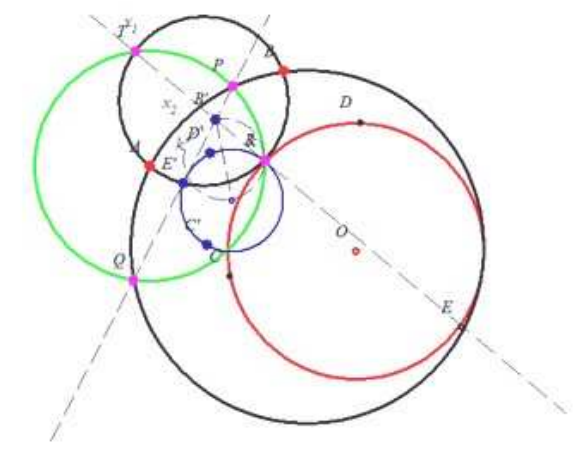

ii) If point $A$ (or point $B$ ) is on the circle $l$, and the other point is outside of the circle, there is only one solution.

iii) If point $A$ (or point $B$ ) is on the circle $l$, and the other point is inside of the circle, there is only one solution.

iv) If both points are inside the circle $l$, there are two solutions.

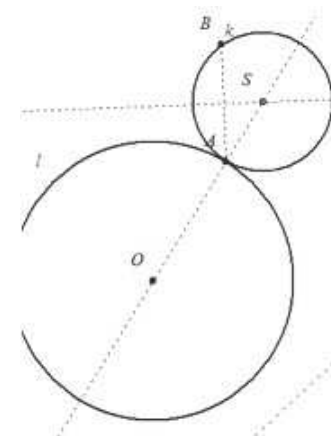

ii)

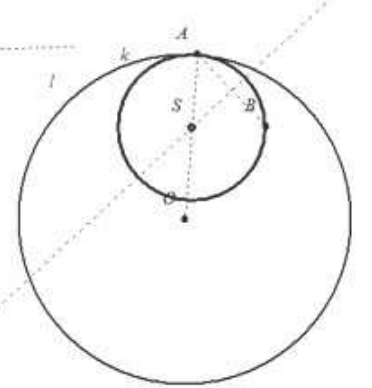

iii)

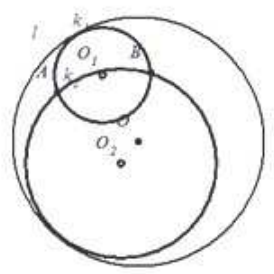

iv) 
(4) CIRCLE ACROSS ONE POINT TANGENTS TWO GIVEN LINES $A, p, q$

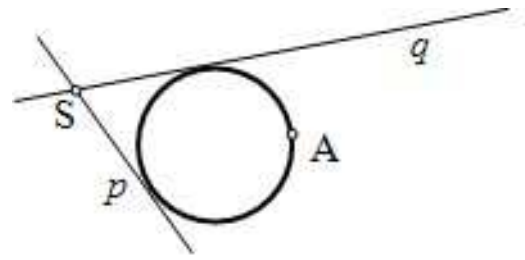

Solution: The center of an arbitrary circle which tangents two lines $p$ and $q$ is on their angle bisector or their symmetry-line. Let $S$ be the intersection point of given lines. Choosing one of these circles, and using dilatations $H_{S, \frac{\overrightarrow{S A}}{\overrightarrow{S B}}}(l)=k_{2}$ and $H_{S, \frac{\overrightarrow{S A}}{\overrightarrow{S C}}}(l)=k_{1}$ with center $S$ and coefficients $\frac{\overrightarrow{S A}}{\overrightarrow{S B}}$ and $\frac{\overrightarrow{S A}}{\overrightarrow{S C}}$, where points $B$ and $C$ are the intersection points of line $S A$ and that chosen circle, there are circles $k_{1}$ and $k_{2}$.
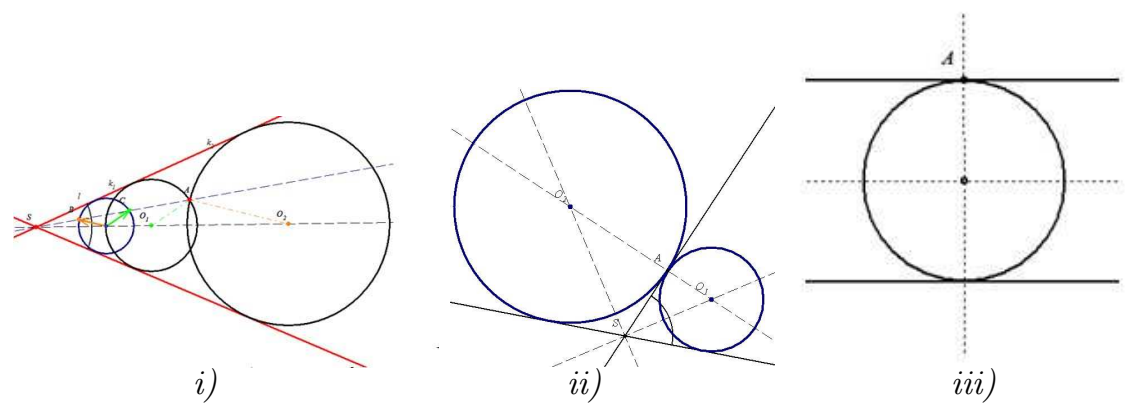

Discussion:

i) If the given lines have a common point or they are parallel and point $A$ isn't on these lines, there are two circles as solution.

ii) If the given lines have a common point, and point $A$ is on one of them, there are two circles as solution.

iii) If the given lines are parallel, and point $A$ is on one of them, then there is only one solution. 


$$
\text { "tmcs-R-Sipos" — 2009/6/2 — 23:53 — page } 77 \text { — \#9 }
$$

(5) CIRCLE ACROSS ONE POINT TANGENTS THE GIVEN LINE AND THE GIVEN CIRCLE $A, p, l$

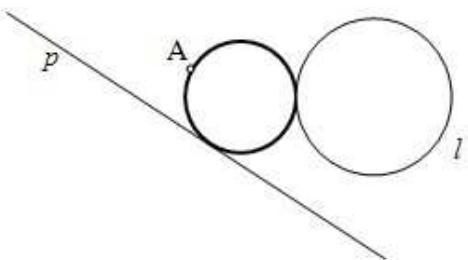

Solution: Using inversion $\psi_{i}(A, r)$ with center $A$ and arbitrary radius $r$ and transforming line $p$ and circle $l$ one can get the images $p^{\prime}$ and $l^{\prime}$ circles. Construct the common tangents $k_{1}^{\prime}, k_{2}^{\prime}, k_{3}^{\prime}$ and $k_{4}^{\prime}$ of these circles. The inverse images of these lines by using again $\psi_{i}(A, r)$ are circles $k_{1}, k_{2}, k_{3}$ and $k_{4}$.

Discussion: If point $A$ isn't either on the circle $l$ nor on the line $p$, there are four solutions of that problem, because in this inversion image of the line $p$ is circle, so two circles without common points have four common tangents, two inside and two outside.

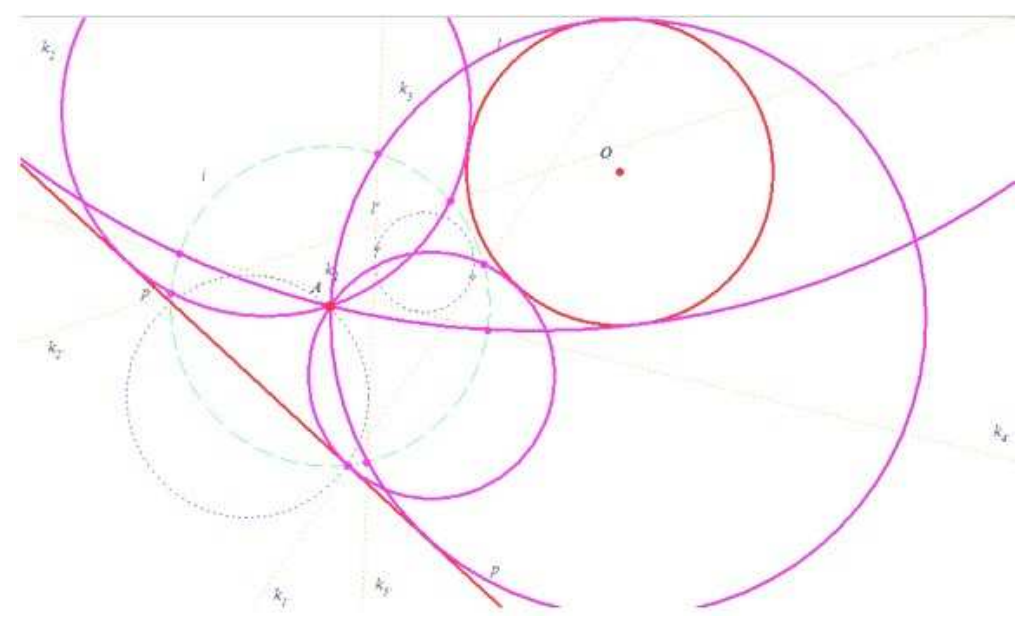

Here DGS (dynamic geometry system) helps to find every possible solution of the problem moving basic elements ( point, circle, line), and replacing them students can see the transformations. In "classical" construction it is almost impossible to find every solution. Drawing with chalk on blackboard rare gives precise construction. Rulers and bows in students' notebook also 
need a great preciosity, although we want to be. My students learn how to construct on paper and by computer, too. Visualization helps in every step of solving the problems: 1 . understanding the task, 2 . construct the solution, 3. control the problem and it's solution.

(6) CIRCLE ACROSS ONE POINT TANGENTS TWO GIVEN CIRCLES A, $l, m$

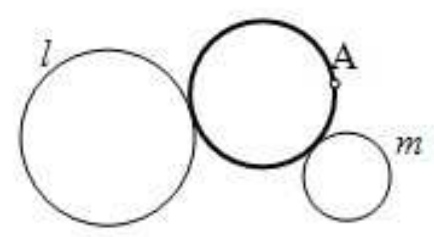

Solution: Using inversion $\psi_{i}(A, r)$ with center $A$ and arbitrary radius $r$ and transforming circles $l$ and $m$ one can get the images, they are circles $l^{\prime}$ and $m^{\prime}$. Construct the common tangents $k_{1}^{\prime}, k_{2}^{\prime}, k_{3}^{\prime}$ and $k_{4}^{\prime}$ of these circles. The inverse images of these lines by using again $\psi_{i}(A, r)$ are circles $k_{1}, k_{2}, k_{3}$ and $k_{4}$.

The step by step construction is bellow:

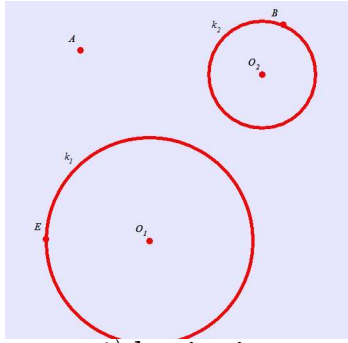

beginning

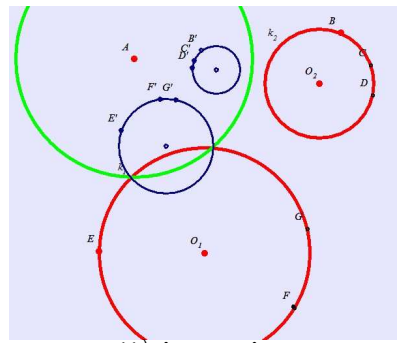

ii) inversion

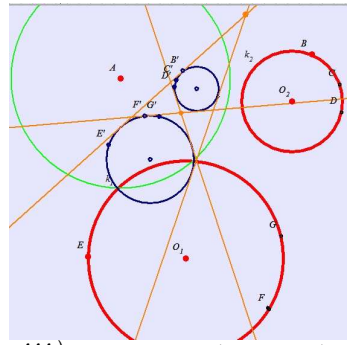

iii) common tangents

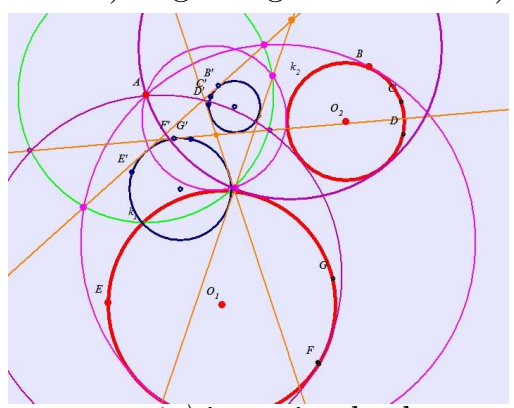

iv) inversion back

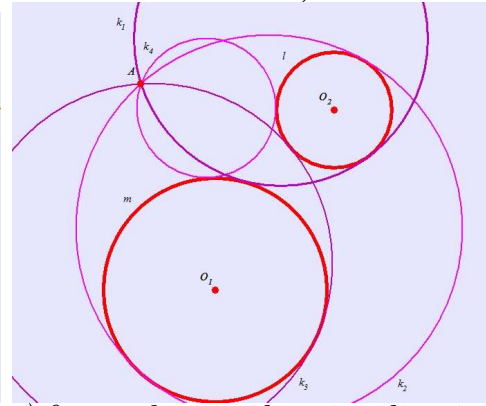

v) four solutions by visualization 
Discussion: If point $A$ isn't either on the circle $l$ nor on circle $m$, there are four solutions of that problem, because in this inversion images of the circles are circles, so two circles without common points have four common tangents, two inside and two outside.

(7) CIRCLE TANGENTS THREE GIVEN LINES $p, q, r$

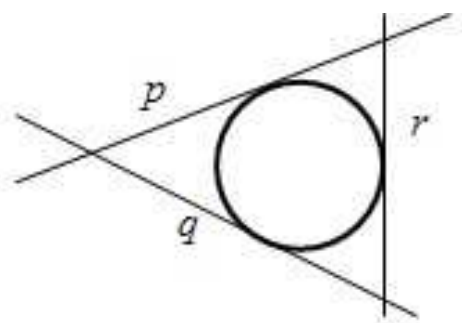

Solution: The solution is the inscribed circle of the triangle if the lines have intersection points in pairs.

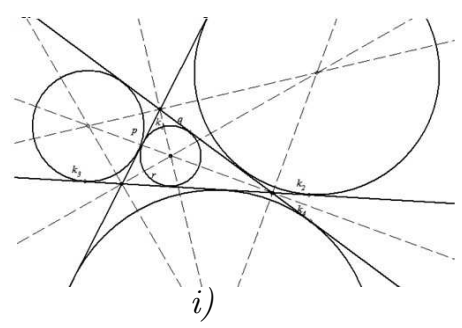

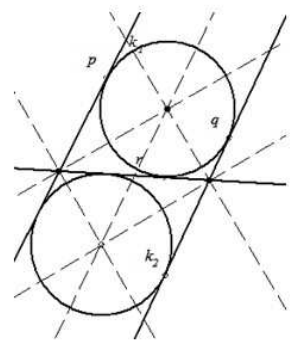

ii)

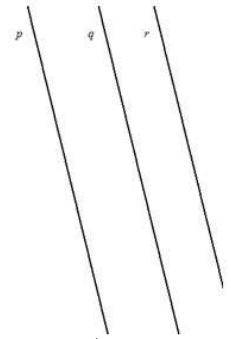

iii)

Discussion: i) If given lines $p, q, r$ form a triangle, the center of this circle is the intersection point of bisectors of this triangle. But there are three circles outside of the triangle. ii) If two lines are parallel and the third line intersects both given lines, there are two circles. iii) If all three lines are parallel, then there isn't any solution of the problem. 
(8) CiRCle tANGENTS TWO GIVEN LINES AND THE GIVEN CIRCLE $p, q, l$

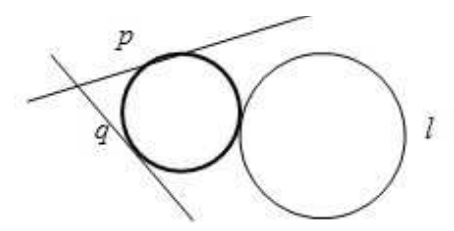

Solution: Every circle which tangents two lines $p$ and $q$ has a center on the symmetry line of these lines. Reduce the given circle $l(O, r)$ to point, decrease the radius $r$ to zero, construct lines $p^{\prime}$ and $q^{\prime}$ parallel to given lines on difference $r$ from given lines and use the solution of the problem 4. After the finding this circle, increase it's radius with $r$. As problem 4 , here exists two solutions, too.

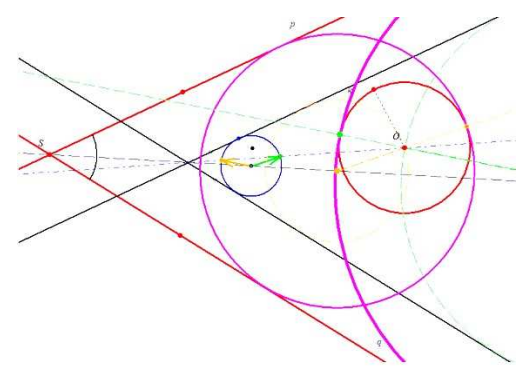

(9) CIRCLE TANGENTS THE GIVEN LINE AND TWO GIVEN CIRCLES $p, l, m$

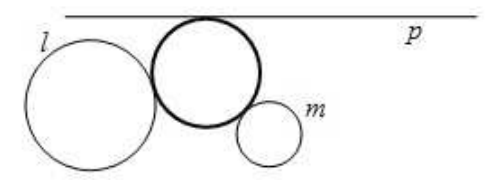

Solution: Let $l\left(O, r_{1}\right)$ and $m\left(S, r_{2}\right)$ be the given circles and let $r_{1}<r_{2}$. Then reduce circle $l$ to point, and reduce circle $m$ 's radius $r_{2}$ with $r_{1}$. Now this problem is equal to problem5. Construct the circle as solution, and increase it's radius with $r_{1}$.

Discussion: If given circles and the line haven't intersection point, there are six possible solutions of the problem. [1] 

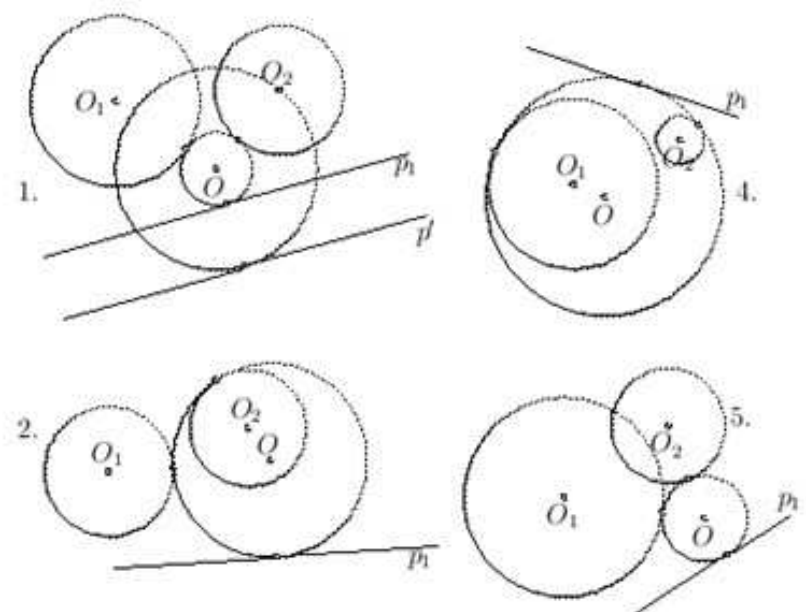

3.
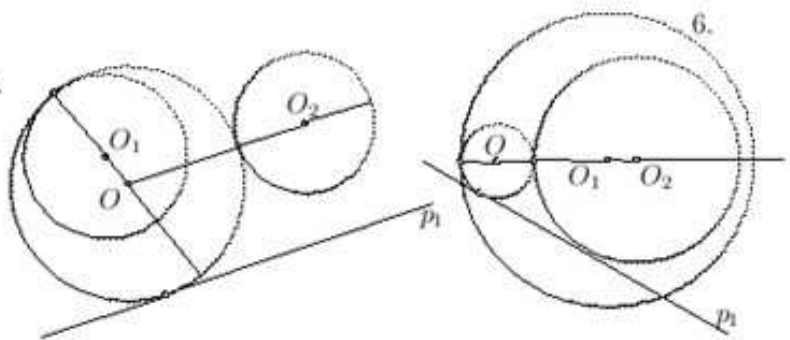

(10) CIRCLE TANGENTS THREE GIVEN CIRCLES $l, m, n$

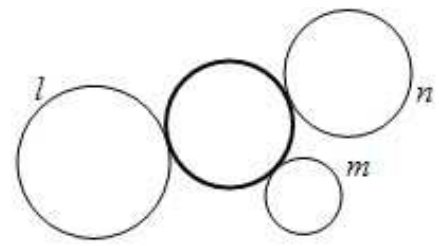

Solution: This problem was the beginning and the most interesting of Apollonius' problems, and later gave the formulation to all of them. Let $m\left(O_{1}, r_{1}\right)$ be the smallest circle. Reducing it to the point, decrease it's radius to zero, and decrease the radiuses $r_{2}$ and $r_{3}$ of circles $l\left(O_{2}, r_{2}\right)$ and $n\left(O_{3}, r_{3}\right)$ with $r_{1}$. Now it is problem6 with one point and two given circles. Find and construct the circle as the solution and increase it's radius with $r_{1}$. 


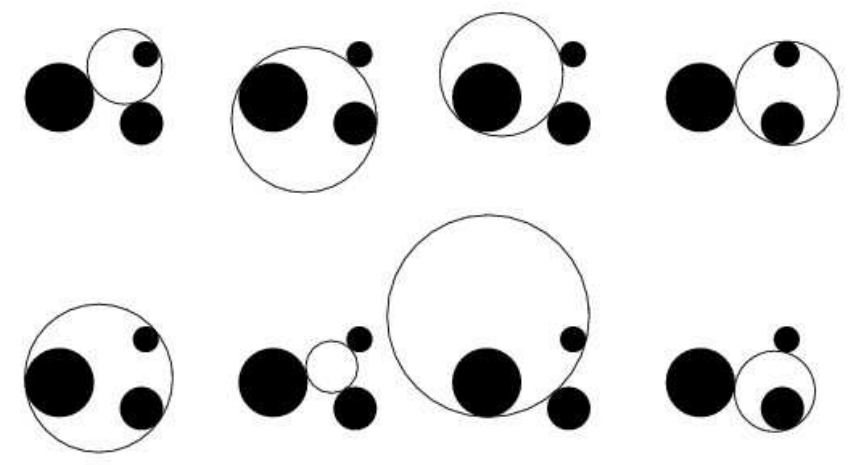

Discussion: If these circles haven't common point, one can get every of eight possible solutions, as bellow from [4].

\section{Results}

Every three months there is a 90-minute-long writing exam in the school, which contains tasks that have been taught in the previous three months, problems to solve and prove. The chosen tasks are from our course book [5], which is translated into Hungarian by me.

Description of researched groups:

Old $=$ control group without visualizations on computers, in 2003; there were 20 students.

$\boldsymbol{N e w}=$ experimental group with 25 percent computer techniques and visualization, in 2004; there were 14 students.

In both of the groups there are students from all over Vojvodina, with different backgrounds, schools and teachers. But all of them are talented at mathematics as all of them have passed the entrance exam, and was accepted in this special grammar school in Hungarian language, which is unique in Serbia.

The results on the first exam were:

\begin{tabular}{|c|c|c|}
\hline & control group & experimental group \\
\hline excellent & 0 & 3 \\
very good & 3 & 3 \\
good & 7 & 1 \\
sufficient & 6 & 7 \\
insufficient & 4 & 0 \\
\hline summary & 20 & 14 \\
\hline average & 2,36 & 3,14 \\
\hline
\end{tabular}


This first exam was the most difficult task in the teaching of axiomatically based theoretical geometry with theorems and their proofs. There is no excellent mark in the control group and the number of the insufficient marks is 4 , which is a very week result. This group learned axioms, theorems and proving without help on imagination, they didn't get impulses any time to solve the more and more difficult tasks. Their dynamism and energy decreased from lesson to lesson. There are 3 excellent marks in the experimental group and there is no insufficient mark. Their average is higher for 0,78. Using DGS on every fourth lesson in IT classroom and visualization for solving the problems in a given tasks, their attention increased and they get more enthusiasm. They solved the difficult and complicated theoretical problems, because they have got a greater self-confidence and pleasure during the lessons of geometry.

The results of the second exam were:

\begin{tabular}{|c|c|c|}
\hline & control group & experimental group \\
\hline excellent & 4 & 3 \\
very good & 4 & 6 \\
good & 7 & 4 \\
sufficient & 4 & 1 \\
insufficient & 0 & 0 \\
\hline summary & 20 & 14 \\
\hline average & 3,42 & 3,79 \\
\hline
\end{tabular}

This exam contained classical tasks from planimetry, and these students are talented at mathematics, that's why there isn't any insufficient mark. Both groups worked diligently, accurately and precisely.

Results of the First Half:

Average grade of the control group is 3,40 .

Average grade of the experimental group is 4,07 .

The results of the third exam were:

\begin{tabular}{|c|c|c|}
\hline & control group & experimental group \\
\hline excellent & 1 & 1 \\
very good & 2 & 1 \\
good & 5 & 1 \\
sufficient & 6 & 7 \\
insufficient & 6 & 3 \\
\hline summary & 20 & 14 \\
\hline average & 2,30 & 2,23 \\
\hline
\end{tabular}

It was the most problematical geometry exam, full of difficult theorems and their proofs, and nice constructions. This was very complicated exam with accurate constructions. The results in the two groups are similar, the difference 
between the average grades is only 0,07 that is almost unimportant. I was very strict in marking the students' works, still they got good marks, which shows that both groups are talented little mathematicians.

The results of the fourth exam were:

\begin{tabular}{|c|c|c|}
\hline & control group & experimental group \\
\hline excellent & 9 & 1 \\
very good & 4 & 7 \\
good & 2 & 3 \\
sufficient & 4 & 1 \\
insufficient & 1 & 0 \\
\hline summary & 20 & 14 \\
\hline average & 3,80 & 3,62 \\
\hline
\end{tabular}

This exam contained tasks also from trigonometry, where visualizations in DGS can't help. The result in the control group is better than in the experimental group. This warns the teacher to be more careful when teaching this part in the future.

\section{The results on the end of the school year:}

These results show and prove the advantage of the experimental group using visualization, any DGS. Average grade of the control group is 3,65. Average grade of the experimental group is 3,93 , which is higher than the previous for 10 percent.

\section{Summary}

In the Bolyai Secondary School, Secondary Grammar School and Dormitory for Gifted Students in Senta I work as a geometry teacher from the beginning of 2003. My method, teaching geometry using visualizations on computer has been applied from 2004. We use "Euklides" DGS that means Dynamic geometry system to help pupils in understanding the difficult problems and constructing the figure with given items. DGS on computer draw figures with special accuracy and one can move basic elements on the figure and follow changes of the figure. In this way one can find out new connections and proof of a given problem. In this way the pupil's motivation increases. They like lessons much more in the IT classroom; they ask for and wait for it. I expect growth of achievement for the end of this year, when our first generation graduates in June. During the first four years of teaching geometry using computers I acquired experience that our pupils at the end of their secondary education receive that kind of tools and knowledge 
which help them to solve problems in their own way with special skills, and to find out new solutions in technical problems as engineer experts. They learn to apply computers and visualizations to their own problems far from geometry and mathematics in every special case of life and their mind is open to new discoveries in the world - as said in the "Mathematical Discovery". Teaching geometry using visualizations on computer prepare the scientist of the future to try every special moment and cases of the new problems to find and discover our environment and life.

\section{References}

[1] A. Bilimović, Apolonijevi problemi, (2007-03-21), www.matf.bg.ac.yu.

[2] Euclid, The Elements, Gondolat kiadó, 1983.

[3] Gy. Hajós, Bevezetés a geometriába, Tankönyvkiadó, Budapest, 1984.

[4] Internet, MathWorld, Wolfram Research, (2007-03-21), http://www . mathworld. wolfram. com.

[5] M. Mitrović, S. Ognjanović, M. Veljković, L. Petković and N. Lazarević, Geometrija I za matematičke gimnazije, Krug Beograd, 2003.

[6] D. Tall, Interrelationships between mind and computer: processes, images symbols, Advanced Technologies in the Teaching of Mathematics and Science, Springer-Verlag, New York, 1993, 385-413.

[7] D. Tall, Enviroments for enactive and visual manipulation, The Psychology of Advanced Mathematical Thinking: Biological Brain and Mathematical Mind, Conference of the International Group for the Psychology of Mathematical Education, Lisbon, July, 1994.

ELVIRA RIPCO SIPOS

BOLYAI GRAMMAR SCHOOL

SENTA

E-mail: siposelvira@bolyai-zenta.edu.rs

(Received January, 2008) 